\title{
Valoración del riesgo de ulcera por presión según la escala de Braden en el paciente neurológico
}

\author{
Ascarruz-Vargas Claudia1, Tapia-Estrada Debbie ${ }^{1}$, Tito-Pacheco Evelyn ${ }^{1}$, Vaiz-Bonifaz Rosa²
}

\section{RESUMEN}

Objetivo: determinar el riesgo de úlceras por presión en el paciente neurológico postrado, mediante la aplicación de la escala Braden en el Servicio de Neurología 13 A del Hospital Nacional Edgardo Rebagliati Martins. Material y métodos: el diseño fue de tipo descriptivo de corte transversal y se realizó durante los meses de octubre a diciembre de 2012, la población estuvo conformada por 120 pacientes postrados al mes y la muestra se obtuvo con la fórmula para estimar proporción poblacional finita, obteniéndose 75 pacientes postrados a través del muestreo aleatorio simple, quienes cumplieron con los criterios de inclusión y exclusión. Para la recolección de datos se utilizó una ficha conjuntamente con la escala de Braden, los datos recolectados fueron procesados y tabulados en una base de datos para el respectivo análisis. Resultados: el estudio mostró que el mayor porcentaje de pacientes evaluados presentaron riesgo moderado con $49 \%$, riesgo bajo con $31 \%$ y alto riesgo con $20 \%$. Conclusiones: la escala Braden, dada su sensibilidad y especificidad, permitió reconocer el nivel de riesgo a úlcera por presión en los pacientes neurológicos.

Palabras clave: Escala Braden, úlceras por presión, paciente neurológico. Perú. (Fuente DeCs BIREME).

\section{Assessing the risk of pressure ulcers according Braden scale in neurological patients}

\begin{abstract}
Objetive: Of this study was to predict the pressure ulcers risk in bedridden neurological patients by using the Braden scale in the Neurology 13 A Edgardo Rebagliati Martins Public Hospital. Material and methods: The design was descriptive cross-sectional and was executed during the months of October to December in 2012, the population consisted by 120 bedridden neurological patients per month and the sample was obtained with the formula for estimating finite population proportion obtaining 75 bedridden patients through simple random sampling, who meet the criteria for inclusion and exclusion. The data was collected by using data collection sheet jointly with the Braden scale, the data collected were processed and tabulated in a database for examination. Results: The study showed that the highest percentage of patients evaluated presented with $49 \%$ moderate risk, low risk with $31 \%$ and $20 \%$ high risk. Conclusions: The Braden scale given its sensitivity and specificity allowed us to recognize the level of pressure ulcer risk in neurological patients.
\end{abstract}

Key words: Braden scale, pressure ulcers, neurological patients. Peru. (Source DeCs BIREME).

\section{INTRODUCCIÓN}

La ocurrencia de úlceras de presión (UPP) en pacientes hospitalizados, es un gran problema de salud que puede ocasionar incomodidad física al paciente, aumento de costos en el tratamiento, necesidad de cuidados intensivos de enfermería, internación hospitalaria prolongada, aumento del riesgo de desarrollo de complicaciones adicionales; necesidad de cirugía correctiva y efectos en la tasa de mortalidad (1). El desarrollo de la UPP es un fenómeno complejo en el que participan varios factores relacionados con el paciente y con el medio externo, siendo la in- 
movilidad el factor de riesgo de mayor importancia en los pacientes hospitalizados (1).

En un estudio sobre uso de la escala de Braden en pacientes hospitalizados en terapia intensiva refieren que el 8,5 y el $26 \%$ representan un problema clínico frecuente en personas hospitalizadas, y más frecuentes en algunas poblaciones como las de adultos mayores, estando asociadas con incremento en los días de estancia hospitalaria, los costos de atención y la mortalidad de los pacientes (2). Los problemas neurológicos comprenden una amplia variedad de trastornos, en las cuales pueden verse comprometidas las funciones de todos los sistemas del organismo humano, los pacientes que cursan con un padecimiento de este tipo, requieren de un amplio conocimiento de la patología de la enfermedad, requiriéndose de una valoración previa específica de cada paciente de acuerdo a la patología, teniendo en cuenta la historia de salud del paciente, antecedentes familiares, antecedentes farmacológicos, el nivel de conciencia, y la función motora (3).

Las úlceras por presión pueden presentarse como complicación de otra patología de base. Así, podemos decir que puede aparecer en pacientes neurológicos con afectación motora, enfermos geriátricos, con grave deterioro de su estado general y con escasa movilidad que les condena a permanecer durante mucho tiempo en la misma posición, pacientes con trastornos del aparato locomotor, con enfermedades crónicas que los incapacitan o con trastornos agudos que precisan extensas férulas o tracciones (4).

Para la identificación de los pacientes que potencialmente pueden padecer este problema es necesario realizar una valoración de los factores de riesgo de ulceración, siendo este el primer paso para la puesta en práctica de los cuidados de prevención (5). Este es un problema que puede prevenirse mediante la atención de enfermería oportuna, segura y continua, ello permitirá la detección a tiempo de pacientes en riesgo de desarrollar úlceras por presión. Es importante disponer de estrategias de educación y prevención integradas dentro de guías de práctica clínica interdisciplinarias que contemplen los distintos niveles asistenciales (6). La prevención es la mejor medida, ya que es utilizado como indicador de la calidad de los cuidados de enfermería (7).

La escala de valoración del riesgo de úlceras por presión (UPP) es una herramienta diseñada para ayudar a identificar a los pacientes que pueden desarrollar una UPP. Las escalas de valoración del riesgo de presentar úlceras por presión (UPP) (EVRUPP) constituyen un instrumento objetivo para valorar y poder tomar decisiones, estando por encima del propio juicio clínico. Su uso sistemático aumentará la calidad de los cuidados que se prodigan al enfermo (5).

Para identificar de forma objetiva y precisa el riesgo de presentar úlceras por presión se han desarrollado diversas escalas. La de Braden es una de las más utilizadas pues evalúa la etiología de las UPP a través de los siguientes caracteres: percepción sensorial, actividad, movilidad, exposición de la piel a la humedad, nutrición y peligro de lesiones cutáneas, con una definición exacta de lo que se debe interpretar en cada uno de los apartados de estos subíndices. Los tres primeros subíndices miden factores relacionados con la exposición a la presión intensa y prolongada, mientras que los otros tres están en relación con la tolerancia de los tejidos a dicha presión. Ha sido validada en diferentes escenarios, tanto en número de estudios como en diferentes medios asistenciales indicando que esta escala ofrece el mejor balance; asimismo, la fiabilidad interobservadores es elevada, de forma que se obtienen resultados consistentes al ser utilizada en distintos medios asistenciales. Es la más adecuada para valorar el riesgo de desarrollar UPP pues presenta la mayor capacidad de predicción y el mejor balance sensibilidad/ especificidad que el resto de escalas (8).

En los servicios de neurología se observan pacientes con distintos factores de riesgo que los hacen vulnerables a desarrollar úlcera por presión por lo cual se ven obligados a permanecer en cama de forma temporal o permanente, perdiendo toda o parte de su autonomía y precisando que otras personas le ayuden a realizar, o realicen por él, las actividades diarias. Este estado de inmovilidad comporta importantes riesgos, ya que gran parte de las funciones vitales precisan de movimiento para realizarse satisfactoriamente. En general, la inmovilidad afecta a todos los sistemas corporales y, cuantas más partes del cuerpo se inmovilicen o más prolongada sea la inmovilización, más graves pueden ser los efectos. Las complicaciones que se asocian a la inmovilidad repercuten en el pronóstico del paciente inmovilizado de forma más intensa, incluso, que la propia enfermedad subyacente. Estas complicaciones aparecen tras periodos cortos de encamamiento; de esta manera están más vulnerables a presentar úlcera por presión si no se les brinda el cuidado indicado y oportuno. Por lo cual, es importante brindar una adecuada atención de enfermería teniendo como prioridad la prevención que se inicia a través de la identificación del riesgo con el uso de una escala que ayudará a objetivar el nivel de riesgo que presente (9). Frente a esta problemática esta investiga- 
ción permitirá diseñar un plan de intervención de enfermería que se basará en la prevención, de manera que se fomente en las enfermeras el uso eficiente de los recursos, y una cultura de prevención en beneficio de la calidad y seguridad del cuidado que brindan al paciente neurológico.

\section{MATERIAL Y MÉTODOS}

Estudio descriptivo de corte transversal. Se realizó en el Servicio de Neurología 13-A del Hospital Edgardo Rebagliati Martins de Essalud en Lima. Los criterios de selección fueron: pacientes del Servicio de Neurología 13A, con hospitalización no mayor de 2 días, con diversos problemas neurológicos, que se encontraron postrados, con discapacidad motora y/o sujeción mecánica permanente. Paciente, familiar o apoderado que firmó el consentimiento informado.

\section{MUESTRA}

Se determinó mediante la fórmula para proporción poblacional finita en la que se consideró un nivel de confianza del $95 \%$ y un error de 5\%. Dando como resultado 75 pacientes seleccionados con la tabla de números aleatorios. Para la recolección de datos se empleó la observación, los instrumentos utilizados fueron la ficha de recolección de datos y la escala Braden.

Para la recolección de datos se planificó los siguientes pasos: la validación del instrumento con una prueba piloto con un total de diez pacientes para determinar la confiabilidad y funcionalidad. El proyecto fue aprobado por el comité institucional de ética de la UPCH. La escala Braden se aplicó al paciente al momento de ingreso al servicio. Se respetó la confidencialidad de los datos y la autonomía del paciente o familiar. Durante la ejecución se contó con el apoyo activo de las enfermeras de turnos del servicio, quienes nos facilitaron la interacción con los pacientes y/o familiares. Los datos fueron procesados utilizando el programa Microsoft Excel 2007 para calcular los porcentajes. Para el análisis estadístico se utilizó la prueba de signo Rango de Wilcoxon para evaluar el cambio en el riesgo de úlcera por presión al inicio y a la semana de evaluación, calculando el chi cuadrado para la correlación de variable.

\section{RESULTADOS}

En la tabla 1 se observa que de los pacientes evaluados el $76 \%$ fueron adultos mayores, el $55 \%$ fueron de sexo femenino, el 58\% fueron casados, el $47 \%$ tuvieron secundaria completa, el $56 \%$ pertenecieron a una familia nuclear y el 77\% presentaron TEC leve.

Tabla 1. Perfil sociodemográfico de los pacientes del Servicio de Neurología 13a - HERM Octubre-diciembre 2012

\begin{tabular}{llrr}
\hline & Perfil sociodemográfico & Total & \% \\
\hline \multirow{3}{*}{ Edad } & Adulto maduro (25-60) & 18 & 24 \\
& Adulto mayor (61- a +) & 57 & 76 \\
& Total & 75 & 100 \\
Sexo & Femenino & 41 & 55 \\
& Masculino & 34 & 45 \\
& Total & 75 & 100 \\
Grado de instrucción & Primaria & 19 & 25 \\
& Secundaria & 35 & 47 \\
& Superior & 21 & 28 \\
& Total & 75 & 100 \\
& Hermanos & 9 & 12 \\
\multirow{2}{*}{ Con quién vive? } & Hijos & 19 & 25 \\
& Nuclear (esposa e hijos) & 42 & 56 \\
& Otros & 5 & 7 \\
& Total & 75 & 100 \\
\hline
\end{tabular}


En la tabla 2 se observa que el riesgo moderado obtuvo el $49 \%$ y el alto riesgo obtuvo el $20 \%$.

Tabla 2. Riesgo a presentar úlcera por presión en los pacientes del Servicio de Neurología 13a-HERM Octubre - diciembre 2012

\begin{tabular}{lcc}
\hline Niveles de riesgo & $\mathbf{N .}^{\mathbf{0}}$ & $\mathbf{\%}$ \\
\hline bajo & 23 & 31 \\
moderado & 37 & 49 \\
Alto & 15 & 20 \\
Total & 75 & 100 \\
\hline
\end{tabular}

En la tabla 3 se observa que en la categoría percepción sensorial predominó la «ligeramente limitada» con el 68\%.

Tabla 3. Nivel de percepción sensorial según escala de Braden en pacientes del Servicio de Neurología 13a - HERM octubre - diciembre 2012

\begin{tabular}{lrr}
\hline \multicolumn{3}{c}{ Percepción sensorial } \\
\hline Categorías & $\mathbf{N .}^{\mathbf{0}^{*}}$ & $\mathbf{\%}$ \\
Completamente limitada & 1 & 1 \\
Muy limitada & 10 & 14 \\
Ligeramente limitada & 51 & 68 \\
Sin limitaciones & 13 & 17 \\
Total & 75 & 100 \\
\hline
\end{tabular}

En la tabla 4 se observa que en la exposición a la humedad predominó la categoría «ocasionalmente húmeda» con el $77 \%$.

Tabla 4. Nivel de exposición a la humedad según escala de Braden en pacientes del Servicio de Neurología 13a-HERM octubre - diciembre 2012

\begin{tabular}{lcc}
\hline \multicolumn{3}{c}{ Exposición a la humedad } \\
\hline Categorías & $\mathrm{N}^{\circ}$ & $\%$ \\
Constantemente húmeda & 1 & 1 \\
A menudo húmeda & 12 & 16 \\
Ocasionalmente húmeda & 58 & 77 \\
Raramente húmeda & 4 & 6 \\
Total & 75 & 100 \\
\hline
\end{tabular}

En la tabla 5 se observa que en el nivel de actividad predominó la categoría «encamado(a)» con el 65\%.

Tabla 5. Nivel de actividad según escala de Braden en pacientes del Servicio de Neurología 13a-HERM

Octubre-diciembre 2012

\begin{tabular}{lrr}
\hline \multicolumn{2}{c}{ Actividad } & \\
\hline Categorías & $\mathrm{N}^{{ }^{\circ}}$ & $\%$ \\
Encamado (a) & 49 & 65 \\
En silla & 23 & 31 \\
Deambula ocasionalmente & 3 & 4 \\
Deambula frecuentemente & - & - \\
Total & 75 & 100 \\
\hline
\end{tabular}

En la tabla 6 se observa que el nivel de movilidad predominó la categoría «muy limitada» con el 57\%.

Tabla 6. Nivel de movilidad según escala de Braden en pacientes del Servicio de Neurología 13a - HERM

Octubre - diciembre 2012

\begin{tabular}{lcc}
\hline \multicolumn{2}{c}{ Movilidad } & \\
\hline Categorías & $\mathbf{N}^{\mathbf{0}}$ & $\mathbf{\%}$ \\
Completamente inmóvil & 21 & 28 \\
Muy limitada & 43 & 57 \\
Ligeramente limitada & 11 & 15 \\
Sin limitaciones & - & - \\
Total & 75 & 100 \\
\hline
\end{tabular}

En la tabla 7 se observa que el nivel nutrición predominó la categoría adecuada con el $67 \%$.

Tabla 7. Nivel nutricional según escala de Braden en pacientes del Servicio de Neurología 13a-HERM Octubre-diciembre 2012

\begin{tabular}{lrr}
\hline \multicolumn{2}{c}{ Nutrición } & \\
\hline Categorías & $\mathbf{N .}^{\mathbf{~}^{\mathbf{0}}}$ & $\mathbf{\%}$ \\
Muy pobre & 1 & 1 \\
Probablemente inadecuada & 24 & 32 \\
Adecuada & 50 & 67 \\
Excelente & - & - \\
Total & 75 & 100 \\
\hline
\end{tabular}

En la tabla 8 se observa que en roce y peligro de lesiones en pacientes predominó la categoría «problema potencial» con el $97 \%$. 
Tabla 8. Peligro de lesiones según escala de Braden en pacientes del Servicio de Neurología 13a-HERM Octubre - diciembre 2012

\begin{tabular}{lcc}
\hline \multicolumn{3}{c}{ Roce y peligro de lesiones } \\
\hline Categorías & $\mathbf{N .}^{\mathbf{0}}$ & $\mathbf{\%}$ \\
Problema & 2 & 3 \\
Problema potencial & 73 & 97 \\
No existe problema & - & - \\
Total & 75 & 100 \\
\hline
\end{tabular}

\section{DISCUSIÓN}

En los resultados de la ficha de recolección de datos (tabla 1) se puede observar lo siguiente: la población que predominó estuvo representado por los adultos mayores con un 76\%; en relación al sexo, predominó el femenino con 55\%; en grado de instrucción predominó secundaria completa con $47 \%$; en relación con quien vive predominaron los que pertenece a una familia nuclear (esposo(a) e hijos) con el 56\%. Los resultados hallados según edad coinciden con los resultados encontrados por Gallo y Pachas sobre el estudio realizado de UPP en el paciente adulto mayor, según escala Norton Padomi 2007, donde el mayor porcentaje pertenece a los adultos mayores que se encuentra en un rango de 80 a 89 años (6). Según Potter los ancianos tienen una mayor incidencia de úlcera por presión. Algunos cambios normales en el envejecimiento de la piel tienen que ver con el aumento de riesgo de las úlceras por presión en los ancianos (5). En otro estudio se encontró que las UPP aparecen en el 3 a $10 \%$ de los pacientes hospitalizados en un momento dado; que la tasa de incidencia de desarrollo de una nueva UPP oscila entre 7,7 y 26,9 \%; y que dos tercios de las UPP que aparecen en hospitales ocurren en pacientes mayores de 70 años (10).

Los resultados de este estudio con respecto al sexo coinciden con el realizado por Pinedo quien encontró que el mayor porcentaje de pacientes fue de sexo femenino (11). En el estudio realizado por Gallo y Pachas se encontró que el 44,7\% cuentan con educación secundaria y que el $48,7 \%$ viven con sus hijos (12). En el presente estudio se halló una coincidencia donde predominó la educación secundaria con $47 \%$, muy por el contrario, en relación con quien vive el paciente el mayor porcentaje habita en una familia nuclear con un 56\%. Según Potter, la familia es el contexto social primario en el que se desarrolla la promoción de la salud y la prevención de la enfermedad. La familia influye considerablemente en las conductas sani- tarias de sus miembros. A su vez, el estado de salud de cada individuo influye en el funcionamiento de la unidad familiar y en su capacidad para conseguir los objetivos (9).

Según la tabla 2, en la población estudiada el riesgo moderado obtuvo el $49 \%$ y el alto riesgo lo obtuvo el $20 \%$. Estos resultados difieren con el estudio realizado por Pinedo donde el riesgo moderado obtuvo un 8,5\%, mientras que el alto riesgo un $11,5 \%$. El organismo humano cambia continuamente con la edad, pero los efectos en un individuo en particular depende de la salud, el estilo de vida, la existencia de factores de estrés y las condiciones ambientales, afectando su capacidad funcional y limitando a su capacidad para vivir independientemente, aumentando el nivel de riesgo a desarrollar UPP (9).

En la tabla 3 se puede encontrar que en percepción sensorial, la categoría que predominó fue «ligeramente limitada» con el 68\%. La percepción sensorial es la capacidad para reaccionar ante una molestia relacionada con la piel, el estado de conciencia de una persona influye en el grado con que perciben e interpretan los estímulos. Cualquier factor que disminuye la conciencia altera la percepción sensorial. Este resultado nos indica que no todos los pacientes evaluados tuvieron la capacidad de reaccionar ante órdenes verbales, comunicar sus molestias o la necesidad de que le cambien de posición o presentaron alguna dificultad sensorial que limitaron la capacidad sentir dolor o malestar en alguna de las extremidades (9).

En la tabla 4, podemos encontrar que «exposición a la humedad» predominó la categoría «ocasionalmente húmeda» con el $77 \%$. La exposición a la humedad es el nivel de la piel expuesta a la humedad, reduciendo la resistencia de la piel. Este resultado nos indica que la piel de los pacientes evaluados estuvo ocasionalmente húmeda, requiriendo un cambio suplementario de ropa de cama aproximadamente una vez al día (9).

En la tabla 5, podemos encontrar que en actividad predominó la categoría «encamado(a)» con el 65\%. La «actividad» es el nivel de actividad física destinada a mejorar la salud. Este resultado nos indica que los pacientes evaluados en su mayoría permanecieron en cama debido a diversas patologías (9).

En la tabla 6 se observa que en movilidad predominó la categoría «muy limitada» con el 57\%. La movilidad es la capacidad para cambiar y controlar la posición del cuerpo, siendo importante para la prevención de úlcera por presión. Este resultado nos indica que los pacientes eva- 
luados ocasionalmente efectuaron ligeros cambios en la posición del cuerpo o de las extremidades (9).

En la tabla 7 se aprecia que en nutrición predominó la categoría «adecuada» con el 67\%. La nutrición cumple un papel relevante en el abordaje de la cicatrización de las heridas. Un buen soporte nutricional no solo favorece la cicatrización de las úlceras por presión sino que también puede evitar la aparición de estas. Este resultado nos indica que los pacientes evaluados, en su mayoría, consumieron más de la mitad de la mayoría de sus alimentos (9).

En la tabla 8 se encuentra que en roce y peligro de lesiones predominó la categoría «problema potencial» con el 97\%. Este resultado nos indica que los pacientes evaluados se movilizaron muy débilmente o requirieron de una mínima asistencia durante los movimientos, la piel probablemente rozó contra parte de las sábanas, silla, sistemas de sujeción u otros objetos (9). Cualquier paciente que experimenta una restricción de la movilidad, alteración funcional neurológica, reducción de la percepción sensorial o reducción circulatoria corre el riesgo de desarrollar UPP. Existe una diversidad de factores que pueden predisponer a un paciente a la formación de UPP. Estos factores pueden estar directamente relacionados con la enfermedad como una reducción del estado de conciencia relacionados con los efectos consecutivos a un traumatismo o secundarios a una enfermedad, como una reducción en la percepción sensorial a consecuencia de un accidente cerebro vascular, estos factores asociados a la humedad y mala nutrición aumentan el riesgo a presentar UPP (9).

Se estima que el $18 \%$ de las personas mayores de 65 años tienen problemas para movilizarse sin ayuda y que a partir de los 75 años las cifras aumentan al 50\%, estando un $20 \%$ confinado en su domicilio $(13,14)$. La disminución en la actividad y la movilidad constituyen indicadores para la dependencia funcional y tienen repercusiones negativas sobre la salud y la calidad de vida (15).

Se concluye que la escala Braden permite valorar condiciones de factores de riesgo (percepción sensorial, humedad, nivel de actividad, movilidad nutrición) para presentar ulceras por presión, sobre todo en pacientes con problemas neurológicos como los del estudio.

\section{REFERENCIAS BIBLIOGRÁFICAS}

1. Magnani L, Larcher M. Uso de la Escala de Braden y de Glasgow para identificar el riesgo de Úlcera Por Presión en pacientes internados en un centro de terapia intensiva. Revista LatinoAmericana Enfermagem 2008; 16 (6).

2. Valdez D, Mena J, Gonzales S. Uso de la escala de Braden en pacientes hospitalizados en terapia intensiva. Revista Metamorfosis en Enfermería del Instituto Nacional de Ciencias Médicas y Nutrición 2011.

3. Álvarez M. Atención de Enfermería al paciente con problemas neurológico. Revista Pisa Farmacéutica 2008.

4. Perez R, Rodriguez E, Escobar A. Frecuencia de úlceras por presión en el paciente lesionado medular, su correlación con el nivel neurológico y escala de ASIA. Revista Mexicana de Medicina Física y Rehabilitación 2008.

5. Arrollo A, Osses B, Garrido R. Riesgo de Aparición de Úlcera Por Presión. Revista Enfermero Red Chilena 2010.

6. Moreno J. las úlceras por presión. España: medbook; 2009.

7. Martinez N, Rodriguez M. Úlcera por Presión y estado nutricional en el paciente grave en el Hospital Naval. Revista Cubana de Medicina Intensiva y Emergencia 2008.

8. Rodríguez M, Soldevilla J. Juicio clínico o escala de valoración para identificar a los pacientes en riesgo de desarrollar úlcera por presión. Rev. Enferm Scielo 2008.

9. Potter P, Perry A. Fundamentos de Enfermería, 5ta ed. Vol II. España: Mosby; 2002.

10. Alfonso C, Baiz J, Lopez L. Cirugía reconstructiva de las úlceras por presión. Rev Cubana 2008.

11. Pinedo J. Aplicación de la escala Braden y presencia de Úlceras por presión en paciente de alto y moderado riesgo-servicio de medicina Hospital Nacional Cayetano Heredia (Tesis). Lima; Universidad Peruana Cayetano Heredia 1999.

12. Gallo K, Pachas Carol. Riesgo a úlceras por presión, según escala Norton Padomi (Tesis) Lima; Universidad Peruana Cayetano Heredia 2007.

13. Lama J. «Síndrome Geriátricos - Características de Presentación de las enfermedades en el adulto mayor». Perú - 2013.

14. Varela L. «Tópicos Selectos en Geriatría». Servicio de Geriatría HNCH: Perú - 2008.

15. OPS, OMS. «Aspectos Clínicos en la Atención de los Adultos Mayores. Proyecto Regional para la Atención Primaria en Salud» 2008.

16. Cerdan E, García K, Pinto L. Valoración del riesgo de úlceras por presión en pacientes adultos y medidas preventivas aplicadas por las enfermeras que laboran 
en el Centro Médico Naval (Tesis) Lima; Universidad Peruana Cayetano Heredia 2001.

17. Arantón L, Rumbo J, Delgado R, Goas R, Sierto E, López N, et al. Prevención de Úlceras por Presión en las unidades de Cuidados Críticos. Cetifac 2008.

18. Saludalia.com. Salud del anciano úlcera por presión. España: saludalia.com 2008.

19. Cantún-UF, Uc-Chi N. Perfil de riesgo para el desarrollo de úlceras por presión en pacientes adultos hospitalizados. Rev. Enferm IMMS 2008.

20. Úlceras y Heridas «comunicación online». Prevención (Monografía). Lima: úlcera y heridas crónicas 2009.

21. Global ActiononAging. Los geriatras consideran una negligencia no adoptar medidas preventivas frente a las úlceras por presión. España, 2009.

\section{Correspondencia}

Rosa Vaiz Bonifaz

Universidad Peruana Cayetano Heredia

Dirección: Av. Honorio Delgado 430, Urb. Ingeniería, S.M.P.

Correo electrónico: rosa.vaiz@upch.pe

Forma de citar este artículo: Ascarruz-Vargas C, Tapia-Estrada D, Tito-Pacheco E, Vaiz-Bonifaz R. Valoración del riesgo de ulcera por presión según la escala de Braden en el paciente neurológico. Rev. enferm Herediana.2014;7(1):10-16. 